\title{
Modeling the winds and magnetospheres of active OB stars
}

\author{
Richard H. D. Townsend
}

Department of Astronomy, University of Wisconsin-Madison, Madison, WI 53706, USA

\begin{abstract}
After briefly reviewing the theory behind the radiative line-driven winds of OB stars, I examine the processes that can generate structure in them; these include both intrinsic instabilities, and surface perturbations such as pulsation and rotation. I then delve into wind channeling and confinement by magnetic fields as a mechanism for forming longer-lived circumstellar structures. With a narrative that largely follows the historical progression of the field, I introduce the key insights and results that link the first detection of a magnetosphere, over three decades ago, to the recent direct measurement of magnetic braking in a number of active OB stars.
\end{abstract}

Keywords. magnetohydrodynamics: MHD, stars: early-type, stars: emission-line, stars: magnetic fields, stars: mass loss, stars: rotation, stars: winds, outflows

\section{Line-Driven Winds}

Stars lose mass in a radiation-driven wind whenever the effective Eddington parameter - representing the ratio of radiative to gravitational acceleration - exceeds unity in their outer layers. In OB stars, however, the pertinent parameter is not the usual

$$
\Gamma_{\mathrm{e}}=\frac{\kappa_{\mathrm{e}} L_{*}}{4 \pi G M_{*} c}
$$

associated with continuum electron-scattering opacity (here denoted by $\kappa_{\mathrm{e}}$ ), but instead that associated with line opacity arising in resonance-scattering transitions. Within the Castor, Abbott \& Klein (1975, hereafter CAK) formalism for these line-driven winds, the effective Eddington parameter is given by

$$
\Gamma_{\ell}=\frac{1}{1-\alpha} \Gamma_{\mathrm{e}} \bar{Q}\left(\frac{\mathrm{d} v / \mathrm{d} r}{\rho c \bar{Q} \kappa_{\mathrm{e}}}\right)^{\alpha}
$$

where the notation follows Owocki (2004), and in particular $\bar{Q}$ is a measure of the total line opacity in the wind, in units of the electron scattering opacity $\kappa_{\mathrm{e}}$ (see Gayley 1995). The term in parentheses is the reciprocal of the Sobolev optical depth; through the appearance of the spatial velocity gradient $\mathrm{d} v / \mathrm{d} r$, it represents the degree to which the wind is able to Doppler-shift the lines out of their own shadow.

The inverse dependence of the radiative acceleration on the density $\rho$ naturally introduces a negative feedback loop that helps to self-regulate the wind. If too much mass is launched from the surface, the acceleration decreases to a point where $\Gamma_{\ell}$ drops below unity; the wind then stalls and falls back to the stellar surface. Accordingly, the mass-loss rate $\dot{M}$ of a radiatively driven wind is not a free parameter, but instead established by this self-regulation process. That is, $\dot{M}$ is an eigenvalue of the system, established by the requirement that a smooth velocity profile links the subsonic outflow at the stellar surface to the supersonic outflow at the nominal outer (far-star) boundary. In the idealized 


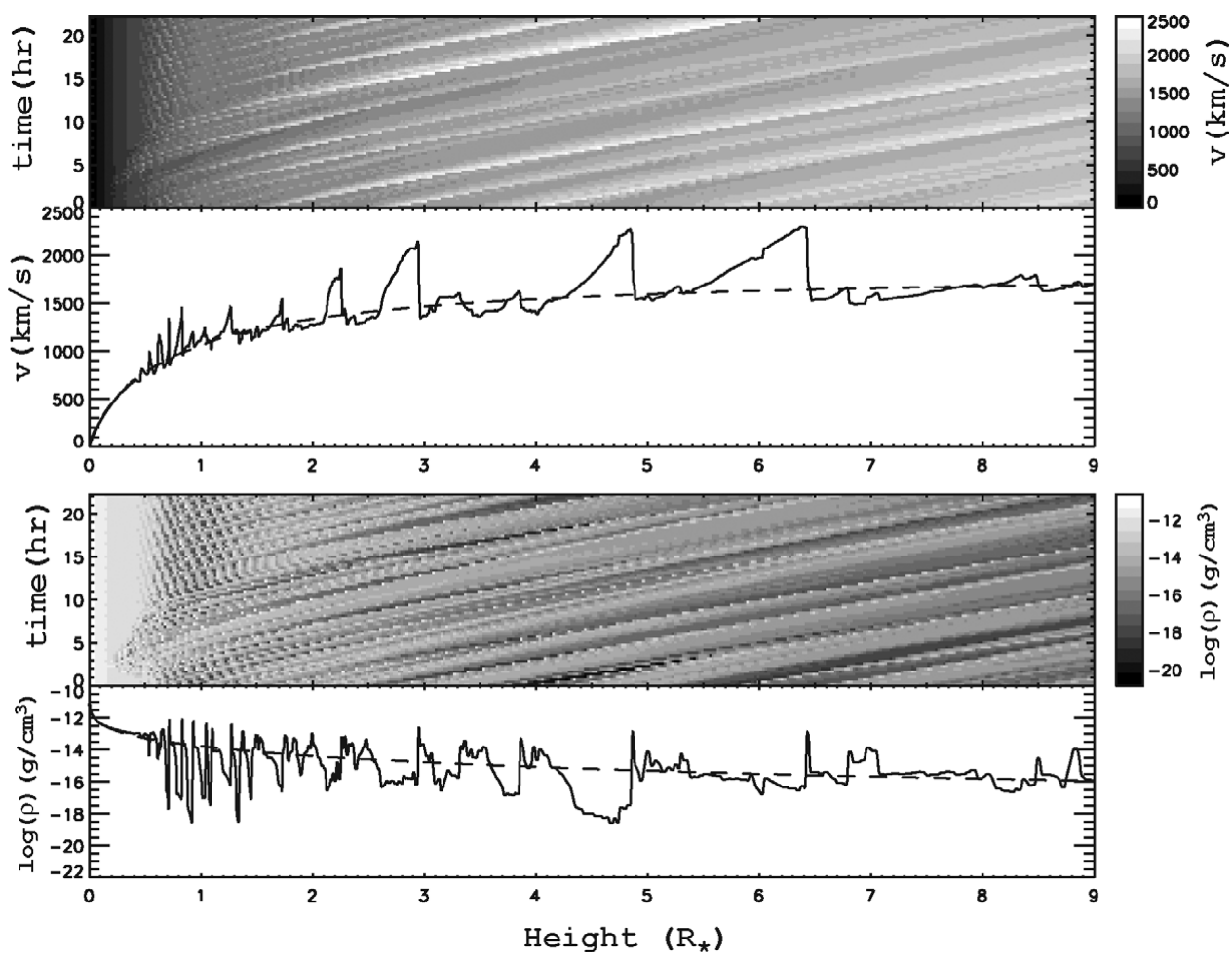

Figure 1. Results of 1-D hydrodynamical simulations of the line-deshadowing instability, calculated using the Smooth-Source-Function formulation of Owocki (1991). The line plots show the spatial variation of velocity (upper) and density (lower) at a fixed, arbitrary time snapshot. The corresponding grey scales show both the time (vertical axis) and height (horizontal axis) evolution. The dashed curve shows the corresponding smooth, steady CAK model (figure courtesy of Stan Owocki).

point-star case, this eigenvalue is given by CAK theory as

$$
\dot{M}_{\mathrm{CAK}}=\frac{L_{*}}{c^{2}} \frac{\alpha}{\alpha-1}\left(\frac{\bar{Q} \Gamma_{\mathrm{e}}}{1-\Gamma_{\mathrm{e}}}\right)^{(1-\alpha) / \alpha},
$$

and solution of the equation-of-motion for $\dot{M}=\dot{M}_{\mathrm{CAK}}$ gives a wind terminal velocity of

$$
v_{\infty}=\sqrt{\frac{\alpha}{1-\alpha} \frac{2 G M_{*}}{R_{*}}} .
$$

The run of wind velocity with radius can be well-approximated by a so-called $\beta$-law,

$$
v(r)=v_{\infty}\left(1-\frac{R_{*}}{r}\right)^{\beta}
$$

where $\beta$ is typically around 0.5 . Owocki (2004) provides an excellent review of the formalism leading to these various results.

\section{Wind Structure}

The CAK theory outlined above presumes a smooth wind outflow from a spherically symmetric star. In reality, we know from observations of OB stars that this cannot be 
the case; there is ample evidence for wind structure at both small and large scales (see, e.g., the review by Alex Fullerton). Where does this structure come from?

A simple linear perturbation analysis of radiative driving (e.g., Lucy \& Solomon 1970) predicts a perturbed line force that is directly proportional to the perturbed velocity; this simply reflects the amount of additional opacity that is Doppler shifted out of its own shadow at the blue edge of line profiles. As a result, line-driven winds are linearly unstable, with small disturbances amplified exponentially. The typical growth timescale is $\tau_{\text {grow }} \sim 10^{-2} \tau_{\text {wind }}$, where $\tau_{\text {wind }}=R_{*} / v_{\infty}$ is the wind flow timescale; therefore, this so-called line-deshadowing instability (LDI) is potentially a very powerful source of wind structure.

A more-detailed analysis indicates that the LDI operates only for velocity perturbations whose physical length scale is shorter than the Sobolev length $\ell_{\mathrm{sob}}=v_{\mathrm{th}} /(\mathrm{d} v / \mathrm{d} r$ ) (see Owocki \& Rybicki 1984). At larger scales, the perturbed line force is proportional to the perturbed velocity gradient (as one might expect from eqn. 1.2), and the wind is stable. Accordingly, the LDI is primarily responsible for the generation of small-scale structure in line-driven winds.

Numerical simulation of the LDI can be computationally expensive, as the Sobolev approximation - which assumes a smooth wind at length scales $<\ell_{\text {sob }}$ - cannot be used. 1-D hydro simulations by Feldmeier \& Owocki (1998) indicate that the instability breaks up a smooth CAK wind solution into a sequence of reverse shocks, where fast, low-density wind material runs into the back of slower-moving, high-density material (see Fig. 1). These wind shocks are considered a likely source for the soft, broad-lined X-ray emission observed in many single OB stars (e.g., Owocki \& Cohen 2006, and references therein). Extending the simulations to 2-D, Dessart \& Owocki (2005) find that the shelllike shocks produced by the LDI are disrupted by Rayleigh-Taylor instabilities, and the wind structure rapidly becomes incoherent down to angular scales approaching the grid scale. Thus, it appears difficult for the LDI to play any significant role in generating large-scale wind structure.

A more plausible origin for this structure is by imprinting from the star itself. OB stars can exhibit inhomogeneities in their surface properties (e.g., temperature, velocity, abundances, magnetic fields), which seed large-scale, non-axisymmetric disturbances in their wind outflows. Rotational modulation of these disturbances seems a promising explanation for cyclical wind variability seen in UV resonance lines of active OB stars, such as episodic blueward-migrating discrete absorption components (DACs; e.g., Kaper \& Henrichs 1994). Prinja et al. (1995) discovered a novel form of variability in the wind of the B0.5Ib supergiant HD 64760, consisting of $1.2 \mathrm{~d}$-periodic absorption modulations superimposed over a longer-timescale DAC pattern. The phase-bowed structure of these modulations led Owocki et al. (1995) to propose that they arise in co-rotating, mutually interacting wind streams rooted in stellar-surface variations. Subsequent work by Cranmer \& Owocki (1996) and Fullerton et al. (1997) fleshed out this idea of co-rotating interaction regions (CIRs; see also Mullan 1984); however, these authors were not able to determine the nature of the surface variability responsible for forming the CIRs in HD 64760. A significant step forward came with the discovery by Kaufer et al. (2006) that the star's wind-sensitive $\mathrm{H} \alpha$ line is variable on a $6.8 \mathrm{~d}$ period, corresponding to the beat period between photospheric non-radial pulsation modes. This represents the first real evidence of the long-sought 'photospheric connection' between surface and wind variability. Nevertheless, the link between this $6.8 \mathrm{~d}$ period and the $1.2 \mathrm{~d}$ CIR period still remains unclear.

Rotation also plays a more-direct role in generating large-scale wind structure, through the action of the centrifugal force. A simple 1-D extension of eqn. (1.3) gives a 

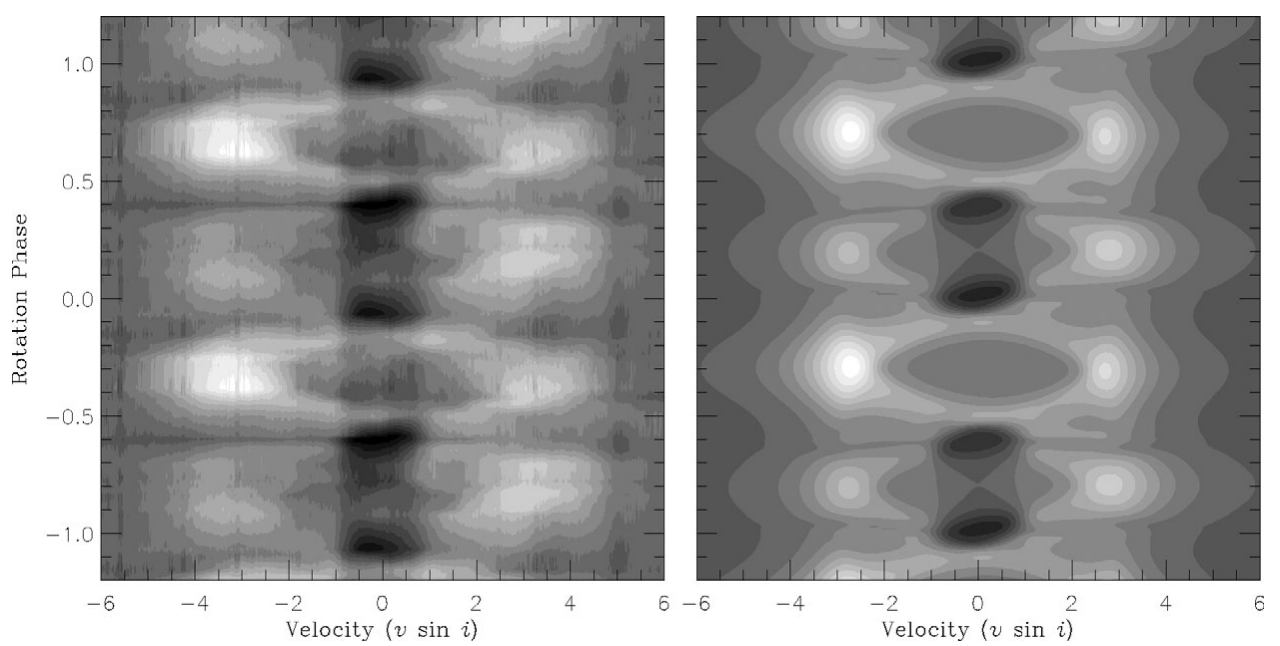

Figure 2. Observed (left) and modeled (right) time-series for the circumstellar $\mathrm{H} \alpha$ emission of $\sigma$ Ori E, phased on the star's $1.2 \mathrm{~d}$ rotation period. White indicates emission relative to the background photospheric profile, and black indicates absorption. The velocity axis is expressed in units of the star's projected rotation velocity, $v \sin i=160 \mathrm{~km} \mathrm{~s}^{-1}$.

latitude-dependent effective mass-loss rate

$$
M_{\mathrm{CAK}}(\theta)=\frac{F R_{*}^{2}}{c^{2}} \frac{\alpha}{\alpha-1}\left(\frac{\bar{Q} \Gamma_{\mathrm{e}}}{1-\Gamma_{\mathrm{e}}}\right)^{(1-\alpha) / \alpha},
$$

where now

$$
\Gamma_{\mathrm{e}}(\theta)=\frac{\kappa_{\mathrm{e}} F}{g_{\mathrm{eff}} c},
$$

and $F$ and $g_{\text {eff }}$ are the local radiative flux and effective gravity, respectively.

A naive interpretation of eqn. (2.1) suggests an equatorially enhanced mass loss (i.e., a disk-like outflow), due to the decrease in $g_{\text {eff }}$ toward the equator, and the consequent increase in $\Gamma_{\mathrm{e}}$. However, as first observed by Cranmer \& Owocki (1995), $\Gamma_{\mathrm{e}}$ is invariant across the surface of a radiative-envelope star, owing to the von Zeipel (1924) gravitydarkening law $F \propto g_{\text {eff }}$. Thus, the bracketed term in eqn. (2.1) does not depend on latitude (although it does depend on rotation rate; see, e.g., Maeder \& Meynet 2000). The latitude dependence of $\dot{M}$ in fact arises via the $F R_{*}^{2}$ term, which is strongest at the poles. Hence, line-driven mass loss from rapidly rotating stars should be bi-polar rather than disk-like. Observational evidence that this is indeed the case continues to mount (e.g., Smith et al. 2003).

Of course, this narrative excludes the possible variation of the opacity parameter $\bar{Q}$ with latitude. The interplay between gravity darkening and the bistability jump can enhance $\bar{Q}$ sufficiently that an equatorially enhanced wind ensues. This has been proposed as a mechanism for forming the dense outflow disks of B[e] stars (e.g., Lamers \& Pauldrach 1991).

\section{Circumstellar Structure}

In addition to transient wind structures that continually advect outward, active OB stars can harbor plasma in circumstellar structures that persist over many wind flow times and/or rotation cycles. Historically, the focus has been on the equatorial Keplerian 
disks of classical Be stars, as discussed in detail in the review by Alex Carciofi. These disks seem unlikely to be formed by wind outflows, because the predominantly radial line driving supplies none of the angular momentum necessary to place material into bound Keplerian orbits.

However, there are other classes of $\mathrm{OB}$ star that exhibit the signatures of persistent circumstellar material. One particularly remarkable object is the helium-strong B2Vpe star $\sigma$ Orionis E, which was discovered by Walborn (1974) to show twin-peaked H $\alpha$ emission, modulated on a $1.2 \mathrm{~d}$ cycle identified with the rotation period (see Fig. 2). Subsequent investigations (see Groote \& Hunger 1982, and references therein) revealed corresponding rotational modulation in a panoply of observables, and their detection of $\mathrm{a} \sim 10 \mathrm{kG}$ dipole magnetic field led Landstreet \& Borra (1978) to conclude that the star's variability arises from 'hot gas [...] trapped in a magnetosphere above the magnetic equator'.

Similar variable $\mathrm{H} \alpha$ emission has been reported in other stars belonging to the Hestrong class; notable recent examples include $\delta$ Ori C (Leone et al. 2010) and HR 7355 (Rivinius, these proceedings; see also the contribution by Bohlender). Among the Hestrong stars that appear not to show emission, many nevertheless exhibit variable UV CIV resonance lines consistent with trapped circumstellar material (see Shore \& Brown 1990). Common to all of the He-strong stars is the presence of a strong (multi-kG), ordered (typically, dipole) magnetic field.

Outside of the He-strong class, magnetospheres have been discovered around a number of other OB stars. These include $\theta^{1}$ Ori C, the first O-type star to be discovered as magnetic (Wade et al. 2006); HD 191612, another magnetic O star with a surprisingly long rotation period of $538 \mathrm{~d}$ (Donati et al. 2006a); the archetypal early-type pulsator $\beta$ Cephei (Henrichs et al. 2000); and $\tau$ Sco, an unusual B0 star known for some time to be a strong, hard X-ray source (Donati et al. 2006b). In fact, all of these stars emit X-rays from their magnetospheres, for reasons discussed in $\S 5$ below.

\section{Rigid-Field Models}

Given the richness of the observational data, it is straightforward to determine empirically that the magnetospheric plasma around $\sigma$ Ori $\mathrm{E}$ is predominantly confined into a pair of co-rotating clouds situated above the intersections between magnetic and rotational equators (e.g., Landstreet \& Borra 1978; Groote \& Hunger 1982). But what is the physical reason for such a plasma distribution? Nakajima $(1981,1985)$ was the first to move successfully beyond phenomenological explanations, by applying a rigid-field formalism originally developed by Michel \& Sturrock (1974) for modeling the Jovian magnetosphere.

As their name implies, rigid-field magnetosphere models rest on the assumption that field lines are completely rigid in the frame of reference that co-rotates with the star. (I demonstrate in $\S 6$ that this is a reasonable assumption for He-strong stars such as $\sigma$ Ori E). The frozen-flux condition of ideal magnetohydrodynamics (MHD) then constrains plasma to flow along fixed trajectories determined by the field topology; in essence, the field lines behave like rigid pipes. Ignoring for the moment any radiative line driving, the only forces capable of accelerating the plasma are pressure gradients and the tangential (field-parallel) components of the gravity and the centrifugal force arising from enforced co-rotation. Acting in tandem, these forces will bring the plasma on any given field line into magnetohydrostatic equilibrium. In the isothermal case at temperature $T$, 

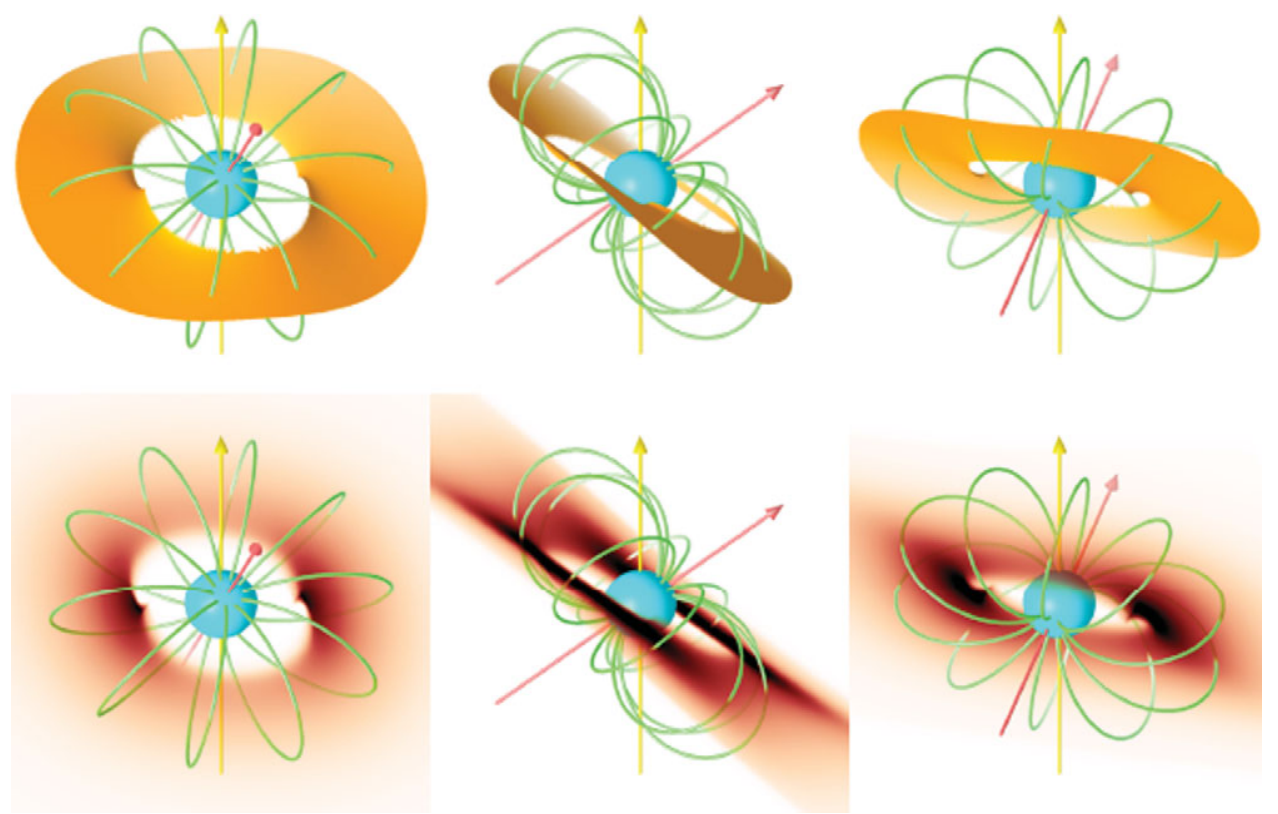

Figure 3. Upper panels: the accumulation surface for a dipole-field star with magnetic obliquity $\beta=55^{\circ}$, viewed from an inclination $i=75^{\circ}$ and at three different azimuths. Although the surface formally extends to infinity, for clarity it has been truncated at a radius $6 R_{*}$. Lower panels: the plasma column density predicted by the RRM model, for the same configurations in the upper panel (although without any truncation). The lower-left panel shows especially well the concentration of plasma into a pair of clouds, situated above the intersections between magnetic and rotational equators.

the equilibrium density distribution is given by the simple expression

$$
\rho(s)=\rho_{0} \exp \left\{-\left[\Phi(s)-\Phi_{0}\right] \mu m_{\mathrm{H}} / k T\right\} .
$$

Here, $s$ is the arc distance measured along the field line from some (arbitrary) reference point $s=0 ; \Phi$ is the effective (centrifugal plus gravitational) potential; and $\rho_{0}$ and $\Phi_{0}$ are the density and effective potential at the reference point. In the frame of reference aligned with the rotation axis, the effective potential in spherical polar coordinates is

$$
\Phi=-\frac{G M_{*}}{r}-\frac{1}{2} \Omega^{2} r^{2} \sin ^{2} \theta
$$

where $\Omega$ is the rotation angular frequency.

Equation (4.1) indicates that the plasma density will be maximal in the vicinity of local minima of $\Phi$, as sampled along a given field line. This is consistent with our physical intuition, which tells us that the plasma will tend to migrate toward the lowest points (i.e., the most negative effective potential). Given that the density drops off exponentially away from the potential minima, the plasma in the magnetosphere will largely be confined to surfaces formed by the loci of the minima. For a dipole field, these so-called 'accumulation surfaces' take the form of warped, tilted disks, whose mean normal lies somewhere between the magnetic and rotational axes (see upper panels of Fig. 3). On the innermost field lines no potential minima exist, because the centrifugal force remains too weak to support plasma against the inward pull of gravity. Therefore, the accumulation surfaces do not extend all the way down to the stellar surface, but are instead truncated 
at a radius commensurate with the Kepler co-rotation radius

$$
R_{\mathrm{Kep}}=\sqrt[3]{\frac{G M_{*}}{\Omega^{2}}}
$$

The beauty of the rigid-field formalism is that it is applicable to completely arbitrary field topologies, and requires extremely modest computational resources. However, because it provides no prescription for how much plasma populates each field line, the normalizing density $\rho_{0}$ remains undetermined. Thus, the rigid-field models of Nakajima and others (e.g., Preuss et al. 2004) are unable to predict the relative distribution of plasma across the accumulation surfaces. As I discuss in $\S 7$, moving past this limitation required insights gleaned from numerical MHD simulations.

\section{The Magnetically Confined Wind Shock Paradigm}

The magnetically confined wind shock (MCWS) paradigm is arguably the most important narrative for understanding the magnetospheres of active OB stars. It was introduced by Babel \& Montmerle (1997a,b) to understand the X-ray emission from IQ Aur and $\theta^{1}$ Ori C. The concept is simple: line-driven wind streams from opposing footpoints of a closed dipole magnetic loop collide at the loop summit, forming standing reverse shocks situated above and below the magnetic equator. Sandwiched between these shocks are regions of hot $\left(\sim 10^{6}-10^{7} \mathrm{~K}\right)$ plasma, which emit X-rays as they cool by radiative recombination; and, in the magnetic equatorial plane, a dense disk-like accumulation of plasma that has had sufficient time to cool back down to near-photospheric temperatures.

Underpinning this narrative is the same rigid-field assumption of earlier models $(\S 4)$. However, a novel element is the recognition that the process filling the magnetosphere is the radiative line driving reviewed in $\S 1$. This is key to being able to model the distribution of plasma throughout the magnetosphere.

As a brief historical aside, the MCWS paradigm was applied to $\theta^{1}$ Ori $\mathrm{C}$ to explain the star's hard ( $\gtrsim 1, \mathrm{keV})$ X-ray emission, before the $\sim 1.1 \mathrm{kG}$ magnetic field was detected. This underscores the notion that the presence of a magnetic field is often signalled through a proxy diagnostic - something to bear in mind, given the high observational cost of direct field detection via spectropolarimetry.

\section{Magnetohydrodynamical Simulations}

The MCWS paradigm was put to the test by ud-Doula \& Owocki (2002) and Owocki \& ud-Doula (2004), who undertook extensive MHD simulations of radiative line-driven winds in the presence of dipole magnetic fields. Beyond confirming the basic wind-shock narrative, these simulations led to a pivotal characterization of the wind/field interaction. Locally, the relative importance of the wind and field in determining flow dynamics can be characterized by the ratio

$$
\eta=\frac{\mathcal{E}_{\text {field }}}{\mathcal{E}_{\text {wind }}}=\frac{B^{2} / 8 \pi}{\rho v^{2} / 2}
$$

between the energy densities $\mathcal{E}$ of the field and the wind. The wind dominates the field when $\eta \ll 1$, and vice versa when $\eta \gg 1$. To determine the overall outcome of this competition, it is useful to write the above expression in the form

$$
\eta(r) \sim \frac{B_{*}^{2} R_{*}^{2}}{\dot{M} v_{\infty}}\left(\frac{r}{R_{*}}\right)^{-2 n} .
$$


This is obtained under the assumption of an $n$-pole field (dipole: $n=2$; quadrupole: $n=$ 3 ; etc.) with strength $B_{*}$ at the stellar surface, together with a wind that is everywhere at the terminal velocity $v_{\infty}$. Based on this expression, ud-Doula \& Owocki (2002) introduced the magnetic confinement parameter

$$
\eta_{*} \equiv \frac{B_{*}^{2} R_{*}^{2}}{\dot{M} v_{\infty}}
$$

as a key determinant of global magnetosphere structure. Substituting this into eqn. (6.2) and setting $\eta(r) \sim 1$ gives an Alfvén radius - at which the flow transitions from fielddominated to wind-dominated — of

$$
R_{\mathrm{Alf}} \sim R_{*} \eta_{*}^{1 / 2 n}
$$

MHD simulations over a range of $\eta_{*}$ values confirm that, qualitatively, field lines remain closed and relatively unperturbed from their force-free configuration in the inner parts of the magnetosphere $\left(r \lesssim R_{\mathrm{Alf}}\right)$, but are ripped open by the wind in the outer parts $\left(r \gtrsim R_{\text {Alf }}\right)$. In the specific case of $\theta^{1}$ Ori $\mathrm{C}$, the simulations reveal a moderately confined wind with field lines closed out to $\approx 2 R_{*}$, consistent with the Alfvén radius for a dipole field $(n=2)$ and a confinement parameter $\eta_{*} \approx 16$.

Although the initial MHD simulations by ud-Doula and Owocki focused on isothermal flow, later calculations incorporate an explicit energy equation to follow the shock heating of material and its subsequent radiative cooling. This allows quantitative predictions of the X-ray emission, suitable for comparison against observations. Focusing again on $\theta^{1}$ Ori C, Gagné et al. (2005a,b) found that the distribution of X-ray-emitting plasma predicted by the non-isothermal MHD simulations is in good agreement with that determined from Chandra HETGS spectroscopy of the star — namely, the bulk of this plasma is situated at $\sim 1.8 R_{*}$, just inside the closed-field regions of the magnetosphere.

\section{The Rigidly Rotating Magnetosphere Model}

In spite of their successes, MHD simulations are not a panacea. They are computationally expensive in 2-D, and even more so in the $3-\mathrm{D}$ required to model systems such as oblique-dipole rotators. Most problematically, the computational cost becomes prohibitive in the limit of large $\eta_{*}$, because the timestep necessary to ensure numerical stability is tiny.

However, in this limit the field lines are almost completely rigid, and the formalism reviewed in $\S 4$ can be applied - if the normalizing density $\rho_{0}$ can be determined. In the rigidly rotating magnetosphere (RRM) model introduced by Townsend \& Owocki (2005), this is done by adopting a result from the MHD simulations by Owocki \& ud-Doula (2004), namely that the stellar-surface mass flux onto a field line tilted by an angle $\Theta$ to the local surface normal is well approximated by

$$
\dot{m}=\frac{\dot{M}_{\mathrm{CAK}}}{4 \pi R_{*}^{2}} \cos \Theta .
$$

The total mass of plasma populating a field line, after a filling time of $\Delta t \dagger$, is $m=$ $\left(\dot{m}_{\mathrm{N}}+\dot{m}_{\mathrm{S}}\right) \Delta t$ (where the subscripts on $\dot{m}$ refer to the Northern- and Southern-hemisphere footpoints). Thus, given the above expression for $\dot{m}$, the normalizing density $\rho_{0}$ can be fixed by requiring that the volume integral of eqn. (4.1) along a field line equal $m$.

$\dagger$ See the Appendix of Townsend \& Owocki (2005), for a discussion of how this filling time might be established. 
For each of accumulation surfaces shown in the upper panels of Fig. 3, the corresponding plasma distributions predicted by the RRM model are shown in the lower panels. As expected, the plasma is confined close to these surfaces, supported against the inward pull of gravity by the centrifugal force. Its distribution across the surfaces is quite non-uniform, however, being concentrated into two clouds situated above the intersections between magnetic and rotational equators - exactly the configuration inferred empirically from the observations.

The RRM model is generally applicable to stars having strong magnetic fields and low mass-loss rates, since these both contribute toward a large $\eta_{*}$ (see eqn. 6.3). Practically speaking, the He-strong stars fall into this category; for instance, the archetype $\sigma$ Ori $\mathrm{E}$ has $\eta_{*} \approx 10^{5}$. Townsend et al. (2005) demonstrate that the RRM model can successfully reproduce the $\mathrm{H} \alpha$ and photometric variability of this particular star (see Fig. 2; also, Oksala, these proceedings). Other stars exhibiting similar $\mathrm{H} \alpha$ emission (e.g., $\delta$ Ori $\mathrm{C}$, HR 7355) seem promising candidates for application of the model, although it is likely that the model will have to be modified to account for certain features such as the time-skewed emission in HR 7355 (see Fig. 4 of Rivinius, these proceedings).

\section{The Rigid-Field Hydrodynamics Approach}

The RRM model focuses on the accumulation of cooled post-shock plasma. Although it incorporates the mass-flux scaling of Owocki \& ud-Doula (2004), it does not provide any explicit description of the wind upflow into the magnetosphere, nor the shocks responsible for heating plasma to X-ray-emitting temperatures. These limitations provided the motivation for Townsend et al. (2007) to develop the rigid-field hydrodynamics (RFHD) approach for dynamical modeling of stellar magnetospheres.

This approach adopts the same rigid-field assumption as the RRM model, but replaces the condition of magnetohydrostatic equilibrium (eqn. 4.1) with hydrodynamical simulations of the wind flow feeding into the magnetosphere from field footpoints. Each field line is treated as a separate 1-D flow system with varying cross-sectional area. Forces acting on the plasma include gravity, pressure gradients, the centrifugal force and the radiative line force. Energy losses due to optically thin radiative cooling and inverse Compton scattering are included, and in more-recent calculations (see Hill \& Townsend, these proceedings) energy transport due to thermal conduction is also modeled. The inclusion of these energetic processes allows predictions of magnetospheric X-ray emission to be made. Therefore, the RFHD approach nicely complements MHD simulations; the latter is valid at small and intermediate values of the confinement parameter $\eta_{*}$, and the former at large values.

Formally, the Alfvén radius is at infinity in RRM and RFHD models. However, in order to avoid overestimating the extent of the magnetosphere (and the amount of emission produced by it), it is necessary to truncate the circumstellar density distribution at the radius where the magnetic and plasma energy densities are equal. The latter is typically dominated not by the kinetic energy of the wind, but by the centrifugal potential energy of the material on the accumulation surfaces. Hence, it is always the case that the truncation radius $R_{\text {trunc }}$ is somewhat less than the (finite) $R_{\text {Alf }}$ defined by eqn. (6.4).

\section{Magnetic Braking}

Recent MHD simulations by ud-Doula et al. $(2008,2009)$ have investigated the interaction between field and wind in rotating, aligned-dipole stars. Toward larger values of 
$\eta_{*}$, the simulations reveal the accumulation of a dense equatorial disk, exactly as predicted by RRM and RFHD models. At the outer, truncation edge of the disk, the field is stretched out into radial configurations by the centrifugally assisted wind. As they advect away from the star, the wind and field carry angular momentum with them, contributing to the gradual braking of the star's rotation.

A key outcome of the ud-Doula et al. (2009) study is a parametrization of the characteristic spin-down timescale $\tau_{\text {spin }}$ due to magnetic braking. For a dipole field,

$$
\tau_{\text {spin }} \sim \tau_{\text {mass }} \frac{3}{2} k \eta_{*}^{-1 / 2}
$$

where $k \approx 0.1$ is a dimensionless measure of the star's moment of inertia, and $\tau_{\text {mass }} \equiv$ $M_{*} / \dot{M}$ is the mass-loss timescale. Applying this expression to $\sigma$ Ori E gives an estimated spin-down timescale of $1.4 \mathrm{Myr}$ - in remarkably good agreement with the direct measurement $\tau_{\text {spin }}=1.34_{-0.09}^{+0.10}$ Myr by Townsend et al. $(2010)$.

The ability for direct comparisons between the theory and observation of magnetic braking is a very exciting development. Amongst non-degenerate objects, active OB stars are unique in this respect; for other types of star, magnetic braking can only be characterized indirectly, by studying populations across a range of ages. However, many uncertainties still remain. For instance, it is not clear how to extend the parametrization above to handle oblique-dipole fields or higher-order fields. With the recent measurement of braking in other active OB stars — in particular, the quadrupole-field HD 37776 (Mikulášek et al. 2008) — the ball is clearly in the theoreticians' court.

\section{The Magnetosphere Zoo}

The magnetospheres of active OB stars can appear like a zoo - the creatures look very different from each other, and it can be difficult to understand how they all derive from a common ancestor. To help dispel any possible confusion, I offer the following 'field guide' for those wanting to know what to expect when searching for or studying magnetospheres:

(i) Does the star have a measured magnetic field?

Yes - go to (ii).

No - either keep looking for a field (example: $\zeta$ Pup), or give up and choose another star.

(ii) Is the confinement parameter $\eta_{*}$ (eqn. 6.3) greater than unity?

Yes - go to (iii).

No - the star's wind is not magnetically confined; the star won't have a magnetosphere (example: $\zeta$ Ori; see Bouret et al. 2008).

(iii) Is the Kepler radius $R_{\text {Kep }}$ (eqn. 4.3) less than the Alfvén radius $R_{\mathrm{Alf}}$ (eqn. 6.4)? Yes - there will be an accumulation of plasma between $R_{\mathrm{Kep}}$ and $R_{\mathrm{Alf}} ; \mathrm{H} \alpha$ emission is a possibility (example: $\sigma$ Ori E).

No - go to (iv).

(iv) Does the star have a large mass-loss rate $\left(\gtrsim 10^{-7} \mathrm{M}_{\odot} \mathrm{yr}^{-1}\right)$ ?

Yes - even though the centrifugal force is insufficient to support plasma, a transient accumulation of plasma out to $R_{\mathrm{Alf}}$ can occur; $\mathrm{H} \alpha$ emission is a possibility (example: $\theta^{1}$ Ori C).

No - the mass-loss rate is too low for any accumulation to occur; no $\mathrm{H} \alpha$ emission is expected (example: $\beta$ Cep; see Favata et al. 2009). 
Having established what to expect, the next step is to choose a suitable modeling approach (e.g., MHD; RRM; RFHD), and attempt to reproduce the observations.

\section{Acknowledgements}

I acknowledge support from NASA grant LTSA/NNG05GC36G.

\section{References}

Babel, J. \& Montmerle, T. 1997a, A\&\&A, 323, 121

Babel, J. \& Montmerle, T. 1997b, ApJ (Letters), 485, L29

Bouret, J.-C., Donati, J.-F., Martins, F., Escolano, C. et al. 2008, MNRAS, 389, 75

Castor, J. I., Abbott, D. C., \& Klein, R. I. 1975, ApJ, 195, 157

Cranmer, S. R. \& Owocki, S. P. 1995, ApJ, 440, 308

Cranmer, S. R. \& Owocki, S. P. 1996, ApJ, 462, 469

Dessart, L. \& Owocki, S. P. 2005, A\&SA, 437, 657

Donati, J.-F., Howarth, I. D., Bouret, J.-C., Petit, P. et al. 2006a, MNRAS, 365, L6

Donati, J.-F., Howarth, I. D., Jardine, M. M., Petit, P. et al. 2006b, MNRAS, 370, 629

Favata, F., Neiner, C., Testa, P., Hussain, G. et al. 2009, A\& $A, 495,217$

Feldmeier, A. \& Owocki, S. 1998, Ap\&SSS, 260, 113

Fullerton, A. W., Massa, D. L., Prinja, R. K., Owocki, S. P. et al. 1997, A\& A, 327, 699

Gagné, M., Oksala, M. E., Cohen, D. H., Tonnesen, S. K. et al. 2005a, ApJ, 628, 986

Gagné, M., Oksala, M. E., Cohen, D. H., Tonnesen, S. K. et al. 2005b, ApJ, 634, 712

Gayley, K. G. 1995, ApJ, 454, 410

Groote, D. \& Hunger, K. 1982, A\&\&A, 116, 64

Henrichs, H. F., de Jong, J. A., Donati, J.-F., Catala, C. et al. 2000, in: M. A. Smith, H. F. Henrichs, \& J. Fabregat (eds.), IAU Colloq. 175: The Be Phenomenon in Early-Type Stars, ASP-CS 214, p. 324

Kaper, L. \& Henrichs, H. F. 1994, ApESSS, 221, 115

Kaufer, A., Stahl, O., Prinja, R. K., \& Witherick, D. 2006, A\& A, 447, 325

Lamers, H. J. G. \& Pauldrach, A. W. A. 1991, A\&A, 244, L5

Landstreet, J. D. \& Borra, E. F. 1978, ApJ (Letters), 224, L5

Leone, F., Bohlender, D. A., Bolton, C. T., Buemi, C. et al. 2010, MNRAS, 401, 2739

Lucy, L. B. \& Solomon, P. M. 1970, ApJ, 159, 879

Maeder, A. \& Meynet, G. 2000, A\&BA, 361, 159

Michel, F. C. \& Sturrock, P. A. 1974, Planetary \& Space Science, 22, 1501

Mikulášek, Z., Krtička, J., Henry, G. W., Zverko, J. et al. 2008, A\&AA, 485, 585

Mullan, D. J. 1984, ApJ, 283, 303

Nakajima, R. 1981, Sci. Rep. Tohoku Univ. Eighth Ser., 2, 130

Nakajima, R. 1985, ApESSS, 116, 285

Owocki, S. P. 1991, in: NATO ASIC Proc. 341: Stellar Atmospheres - Beyond Classical Models (Dordrecht, D. Reidel Publishing Co.), p. 235

Owocki, S. 2004, in: M. Heydari-Malayeri, P. Stee, \& J.-P. Zahn (eds.), EAS Publications Series 13, p. 163

Owocki, S. P. \& Rybicki, G. B. 1984, ApJ, 284, 337

Owocki, S. P., Cranmer, S. R., \& Fullerton, A. W. 1995, ApJ (Letters), 453, L37

Owocki, S. P. \& ud-Doula, A. 2004, ApJ, 600, 1004

Owocki, S. P. \& Cohen, D. H. 2006, ApJ, 648, 565

Preuss, O., Schüssler, M., Holzwarth, V., \& Solanki, S. K. 2004, A\&A A, 417, 987

Prinja, R. K., Massa, D., \& Fullerton, A. W. 1995, ApJ (Letters), 452, L61

Shore, S. N. \& Brown, D. N. 1990, ApJ, 365, 665

Smith, N., Davidson, K., Gull, T. R., Ishibashi, K. et al. 2003, ApJ, 586, 432

Townsend, R. H. D. \& Owocki, S. P. 2005, MNRAS, 357, 251

Townsend, R. H. D., Owocki, S. P., \& Groote, D. 2005, ApJ (Letters), 630, L81

Townsend, R. H. D., Owocki, S. P., \& Ud-Doula, A. 2007, MNRAS, 382, 139 
Townsend, R. H. D., Oksala, M. E., Cohen, D. H., Owocki, S. P. et al. 2010, ApJ (Letters), 714, L318

ud-Doula, A. \& Owocki, S. P. 2002, ApJ, 576, 413

Ud-Doula, A., Owocki, S. P., \& Townsend, R. H. D. 2008, MNRAS, 385, 97

Ud-Doula, A., Owocki, S. P., \& Townsend, R. H. D. 2009, MNRAS, 392, 1022

Wade, G. A., Fullerton, A. W., Donati, J.-F., Landstreet, J. D. et al. 2006, A\&A A, 451, 195

Walborn, N. R. 1974, ApJ (Letters), 191, L95

von Zeipel, H. 1924, MNRAS, 84, 665 